\title{
The Clinical Characteristics and Treatment Response of Patients with Low Grade Non-Hodgkin Lymphoma
}

\author{
Ahmed Yosry El-Agamawi, Mohammed El-Sayed Abd-El-Ftattah Mansy, \\ Mohamed Omar Ahmed*
}

Department of Clinical Oncology and Nuclear Medicine, Faculty of Medicine, Al-Azhar University, Cairo, Egypt

* Corresponding author: Mohamed Omar Ahmed, E-mail: dr.omar_77@yahoo.com

\begin{abstract}
Background: as the histopathologic diagnosis of Non-Hodgkin Lymphoma (NHL) has become more sophisticated with the use of immunologic and genetic techniques, the understanding and treatment of many of the previously described pathologic subtypes have changed. Aim of the Work: was to review the clinicopathologic characteristics of the NHL patients, to assess the treatment response to the different treatment modalities available at our center and to analyze the correlation between patient's characteristics, treatment modality and the achieved response.
\end{abstract}

Patients and Methods: the current work was a retrospective study reviewing the data of patients with pathologically confirmed diagnosis of low-grade NHL referred to Al Hussein university Hospital, Department of Clinical Oncology, Faculty of Medicine, Al-Azhar University during the period between January 2012 till December 2016.

Results: more than half the study group (57.2\%) had the age between 41 and 60 years. Only $14.2 \%$ of the study group was younger than 40 years and $28.6 \%$ were older than 60 years. There was no difference between patients with CLL and patients with Low grade NHL as regards the age distribution. NHL in this study was equal among both males and females in both above 40 and below 40 age groups, unlike what was mentioned before which stated that NHL is more common in males than in females.

Conclusion: only 31 patients were evaluable for survival analysis with median follow up period of 15 months. Median disease-free survival (DFS) was 12 months (95\% CI 0-72.9).

Keywords: Clinical Characteristics, Non-Hodgkin Lymphoma

\section{INTRODUCTION}

As the histopathologic diagnosis of NHL has become more sophisticated with the use of immunologic and genetic techniques, the understanding and treatment of many of the previously described pathologic subtypes have changed. According to the WHO modification of the REAL classification indolent lymphoma/leukemia includes follicular lymphoma, chronic lymphocytic leukemia, lymphoplasmacytic lymphoma, marginal zone B-cell lymphoma, hairy cell leukemia ${ }^{(\mathbf{1 , 2})}$.

Indolent NHL types have a relatively good prognosis with a median survival as long as 20 years, but they usually are not curable. Although they are responsive to different treatment modalities, a continuous rate of relapse is usually seen. Therapeutic options include watchful waiting; rituximab, an antiCD20 monoclonal antibody, alone or with purine nucleoside analogs; oral alkylating agents; and combination chemotherapy Radiolabeled monoclonal antibodies, vaccines, and autologous or allogeneic bone marrow or peripheral stem cell transplantation are also under clinical evaluation Currently, no randomized trials have mature results to guide clinicians about the best options. On a comparative basis, it is difficult to prove benefit when relapsing disease is followed with watchful waiting, or when the median survival is more than 10 years ${ }^{(3,4,5)}$.

Moreover, patients with indolent lymphoma may experience a relapse with a more aggressive histology. Documentation of conversion to a more aggressive histology requires an appropriate change to a therapy applicable to that histologic type. The risk of histologic transformation was $30 \%$ by 10 years in a retrospective review of 325 patients from diagnosis between 1972 and $1999^{(6,7)}$. 
Despite significant improvements in response rates and progression-free survival for patients with low grade lymphoma has been achieved with the addition of rituximab to chemotherapy patients continue to relapse or become refractory to treatment resulting in limited treatment options. Recently, Bendamustine and Obinutuzumab has been shown to be effective in rituximab-refractory patients. Studies demonstrated significantly longer progression-free survival for protocols containing the combination of both agents in this difficult to treat patient group. Moreover, greater proportion of patients reported. The delayed time to worsening with meaningful improvement in health-related quality of life when these drugs are used in combination suggesting that benefit in progression-free survival is not at the expense of an increase in treatment-related toxicity that could lead to reduced health-related quality of life ${ }^{(\mathbf{8})}$.

Indolent lymphoma comprises a unique and challenging subset of non-Hodgkin lymphoma Clinicians continue to face tough choices in its management. However. well planned clinical trials may help in determining how to best manage the clinical continuum that is indolent non-Hodgkin lymphoma ${ }^{(9)}$.

The aim of the current work was to review the clinicopathologic characteristics of the NHL patients, to assess the treatment response to the different treatment modalities available at our center and to analyze the correlation between patient's characteristics, treatment modality and the achieved response.

\section{PATIENTS AND METHODS}

This retrospective study included patients with pathologically confirmed diagnosis of low grade NHL /CLL referred to Al Hussein University Hospital, Department of Clinical Oncology, Faculty of Medicine, AlAzhar University. Approval of the ethical committee and a written informed consent from all the subjects were obtained. This study was conducted between January 2012 till December 2016.
Patients included in the current study should fulfill the following eligibility criteria:

- Age between (16) and (80) years old.

- Performance status (0 to III) WHO.

The files of patients included in the current study were reviewed as regards the: Age, Sex, performance status at presentation, presenting symptom and duration between symptom to diagnosis and presence of comorbidity

All patients included in the study were examined clinically and subjected to a series of laboratory and radiologic investigations. The files of the study group were reviewed as regards the:

- LDH level

- Results of differential blood film and bone marrow examination

- Results of histopathologic examination and immunophenotyping in any

- The site of disease involvement nodal (supra and/or infradiaphragmatic) or extranodal.

- Prevalence of bulky disease among the study group.

- Stage grouping for both low grade NHL according to Ann Arbor staging system $(1,2,3,4)$ and CLL according to Rai staging system $(1,2,3,4)$

The management of patients included in the study group were reviewed. According to the international recommendations for low grade NHL / CLL, patients in the current study with who had no symptomatology were kept under follow up. The clinical characteristics of patients who were kept under follow-up were analyzed.

Patients who had to be treated were treated by either CVP, CHOP or Fludarabine and cyclophosphamide regimens.

Table (1): Chemotherapy regimens used in the current study

\begin{tabular}{|l|c|c|c|c|}
\hline & Drug & Dose & Rout & Days \\
\hline
\end{tabular}




\begin{tabular}{|c|c|c|c|c|}
\hline $\begin{array}{l}\text { CVP } \\
\text { C } \\
\mathrm{V} \\
\mathrm{P}\end{array}$ & $\begin{array}{c}\text { cyclophosphamide } \\
\text { Vicrestin } \\
\text { Prednilon } \\
\end{array}$ & $\begin{array}{c}750 \mathrm{mg} \\
1.4 \mathrm{mg} \\
40 \mathrm{mg} \mathrm{d} 1 \_5 \\
\end{array}$ & $\begin{array}{l}\text { iv } \\
\text { iv } \\
\text { oral }\end{array}$ & $\begin{array}{l}21 \\
21 \\
21 \\
\end{array}$ \\
\hline $\begin{array}{c}\text { CHOP } \\
\mathrm{C} \\
\mathrm{H} \\
\mathrm{O} \\
\mathrm{P} \\
\end{array}$ & $\begin{array}{c}\text { cyclophosphamide } \\
\text { doxurubcin } \\
\text { Vicrestin } \\
\text { Prednilon } \\
\end{array}$ & $\begin{array}{c}750 \mathrm{mg} \\
50 \mathrm{mg} \\
1.4 \mathrm{mg} \\
40 \mathrm{mg} \mathrm{d} 1 \_5\end{array}$ & $\begin{array}{l}\text { Iv } \\
\text { Iv } \\
\text { iv } \\
\text { oral }\end{array}$ & $\begin{array}{l}21 \\
21 \\
21 \\
21 \\
\end{array}$ \\
\hline $\begin{array}{l}\text { Fludarabin/cyclophosphamide } \\
\text { F } \\
\text { C }\end{array}$ & $\begin{array}{c}\text { Fludarabin } \\
\text { cyclophosphamide }\end{array}$ & $\begin{array}{c}25 \mathrm{mg} \mathrm{d} 1 \_3 \\
250 \mathrm{mg} \mathrm{d} 1 \_3\end{array}$ & $\begin{array}{l}\text { Iv } \\
\text { iv }\end{array}$ & $\begin{array}{l}28 \\
28\end{array}$ \\
\hline
\end{tabular}

The clinical characteristics of analyzed.

Patients who received treatment were

All patients were followed up regularly to assess the treatment response and toxicity.

\section{Statistical analysis}

Survival interval will be the time between the date of histological diagnosis and the date of the last follow-up (for censored observations) or the date of death (for uncensored observations), while disease free interval will be the time between date of the first treatment and the date of the last follow-up (for censored observations) or, date of death or disease progression whichever happen first (for uncensored observations).

One-sided log-rank of Kaplan-Meier survival estimates will be used for statistical analysis of overall survival and disease-free survival, while the unpaired T test and one-way ANOVA test were used in the univariate analysis of the variables.

\section{RESULTS}

The current retrospective study included 40 patients with pathologically confirmed diagnosis of low grade NHL /CLL referred to our center during the period between January 2012 till December 2016. During the period of the study, our center accepted 4588 new cases of malignant disease. Malignant lymphoma was diagnosed in 326 cases, 199 patients with aggressive NHL, 40 cases with low grade NHL / CLL and 87 with HD. The incidence of low grade malignant lymphoma to total malignant cases was $0.9 \%$ and to cases with malignant lymphoma was $12.3 \%$ Figure (5) The files of 5 patients had no enough data, therefore, these 5 cases were excluded from the study. The remaining 35 patients constituted the study group.

Table (2): Age distribution of the study group

\begin{tabular}{|c|c|c|c|c|c|c|c|}
\hline \multirow{2}{*}{ Age in years } & \multicolumn{2}{|c|}{ Low grade NHL } & \multicolumn{2}{|c|}{ CLL } & \multicolumn{2}{|c|}{ Total } & $P$ value \\
\hline & No. & $\%$ & No. & $\%$ & No. & $\%$ & \multirow{3}{*}{0.227} \\
\hline Range & \multicolumn{2}{|c|}{$18-78$} & \multicolumn{2}{|c|}{$23-75$} & \multicolumn{2}{|c|}{$18-78$} & \\
\hline Median & \multicolumn{2}{|c|}{33} & \multicolumn{2}{|c|}{31} & \multicolumn{2}{|c|}{33} & \\
\hline$\leq 40$ & 4 & 15.3 & 1 & 11.1 & 5 & 14.2 & \multirow{5}{*}{0.991} \\
\hline $41-50$ & 8 & 30.7 & 3 & 33.3 & 11 & 31.4 & \\
\hline $51-60$ & 7 & 27 & 2 & 22.2 & 9 & 25.8 & \\
\hline $61-70$ & 5 & 19 & 2 & 22.2 & 7 & 20 & \\
\hline$>70$ & 2 & 8 & 1 & 11.1 & 3 & 8.6 & \\
\hline Total & 26 & 100 & 9 & 100 & 35 & 100 & \\
\hline
\end{tabular}

The median age of the whole study group was 33 years. More than half the study group (57.2\%) had the age between 41 and 60 years. Only $14.2 \%$ of the study group were younger than 40 years and $28.6 \%$ were older than 60 years. There was no difference between patients with CLL and patients with Low grade NHL as regards the age distribution. 
The Clinical Characteristics and Treatment Response of Patients...

Table (3): Presenting symptoms of the study group

\begin{tabular}{|l|c|c|c|c|c|c|c|}
\hline \multirow{2}{*}{$\begin{array}{c}\text { Presenting } \\
\text { Symptom }\end{array}$} & \multicolumn{2}{|c|}{ Low grade NHL } & \multicolumn{2}{c|}{ CLL } & \multicolumn{2}{c|}{ Total } & \multirow{2}{*}{ P value } \\
\cline { 2 - 8 } & No. & $\mathbf{\%}$ & No. & \% & No. & \% & \\
\hline Swelling & 14 & 53.8 & 5 & 55.6 & 19 & 54.3 & 0.956 \\
\hline Localized pain & 6 & 23.1 & 2 & 22.2 & 8 & 22.9 & 0.928 \\
\hline General Symptoms & 1 & 3.8 & 2 & 22.2 & 3 & 8.6 & 0.089 \\
\hline Others * & 5 & 19.3 & 0 & 0 & 5 & 14.3 & 0.155 \\
\hline Total & 26 & 100 & 9 & 100 & 35 & 100 & \\
\hline
\end{tabular}

More than half of the study group presented by swelling. This was the same for patients with low grade and those with CLL. However, general symptoms as fever, fatigue, and general weakness were more frequently encountered in patients with CLL.

Table (4): Prevalence of Co-morbidity among the study group

\begin{tabular}{|l|c|c|c|c|c|c|c|}
\hline \multirow{2}{*}{ Co-morbidity } & \multicolumn{2}{|c|}{ Low grade NHL } & \multicolumn{2}{c|}{ CLL } & \multicolumn{2}{c|}{ Total } & P value \\
\cline { 2 - 8 } & No. & \% & No. & \% & No. & \% & \\
\hline Present & 10 & 38.5 & 6 & 66.7 & 16 & 45.7 & \multirow{2}{*}{0.143} \\
\hline Absent & 16 & 61.5 & 3 & 33.3 & 19 & 54.3 & \\
\hline Hypertension & 6 & 23.1 & 4 & 44.4 & 10 & 28.6 & 0.221 \\
\hline Diabetes & 6 & 23.1 & 5 & 55.6 & 11 & 31.4 & 0.07 \\
\hline Ischemic heart disease & 4 & 14.4 & 1 & 11.1 & 5 & 14.3 & 0.751 \\
\hline Positive Hepatitis C markers & 1 & 3.8 & 1 & 11.1 & 2 & 5.7 & 0.418 \\
\hline Others & 0 & 0 & 2 & 22.2 & 2 & 5.7 & 0.013 \\
\hline Total & 26 & 100 & 9 & 100 & 35 & 100 & \\
\hline
\end{tabular}

Co-morbidity was more frequently encountered in patients with CLL than patients with low grade NHL (66.7\% versus 38.5\%, respectively). However, this difference is not statistically significant.

Table (5): Immunophenotyping confirmation of the histopathologic diagnosis for patients in the study group

\begin{tabular}{|c|c|c|c|c|c|c|c|}
\hline \multirow{2}{*}{ Diagnosis confirmed by IPT } & \multicolumn{2}{|c|}{ Low grade NHL } & \multicolumn{2}{|c|}{ CLL } & \multicolumn{2}{|c|}{ Total } & \multirow{2}{*}{$P$ value } \\
\hline & No. & $\%$ & No. & $\%$ & No. & $\%$ & \\
\hline Yes & 12 & 46 & 6 & 66.7 & 18 & 51.1 & \multirow{2}{*}{0.288} \\
\hline No & 14 & 54 & 3 & 33.3 & 17 & 48.6 & \\
\hline Total & 26 & 100 & 9 & 100 & 35 & 100 & \\
\hline
\end{tabular}

All patients were subjected to differential blood count and bone marrow examination. All patients with low grade NHL and 5 patients (5/9 - 55.6\%) of CLL patients had lymph node biopsy for histopathologic examination. In half of the study group the diagnosis was confirmed by immunophenotyping. The distribution of the histopathologic subtypes of cases of low-grade NHL.

Table (6): The clinical characteristics of patient kept under follow up

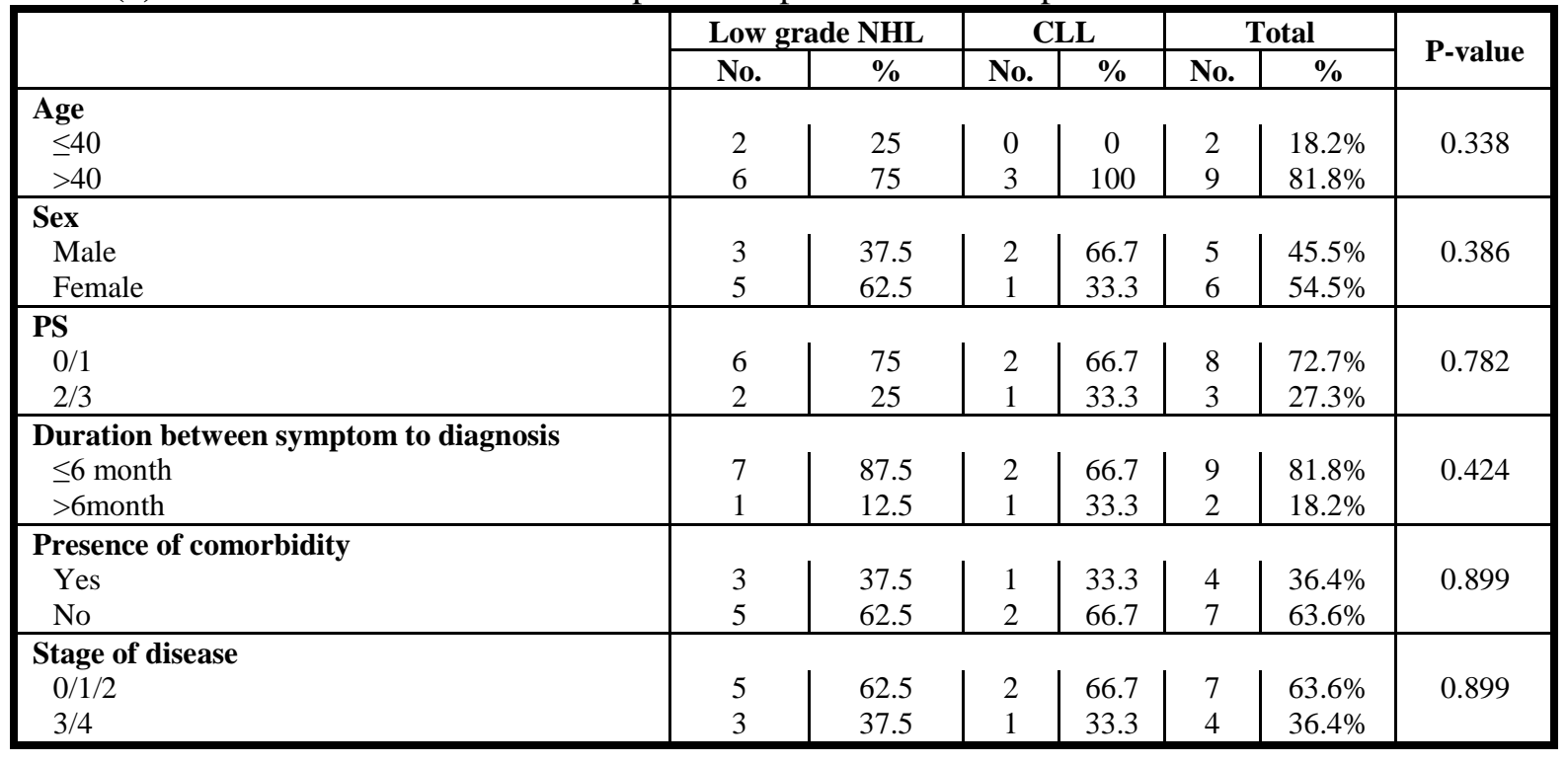


The clinical characteristics of these 11 patients. It was noticed that the majority of patients who were kept under follow-up had early stage of disease.

Table (7): The clinical characteristics of patient received combination chemotherapy at presentation

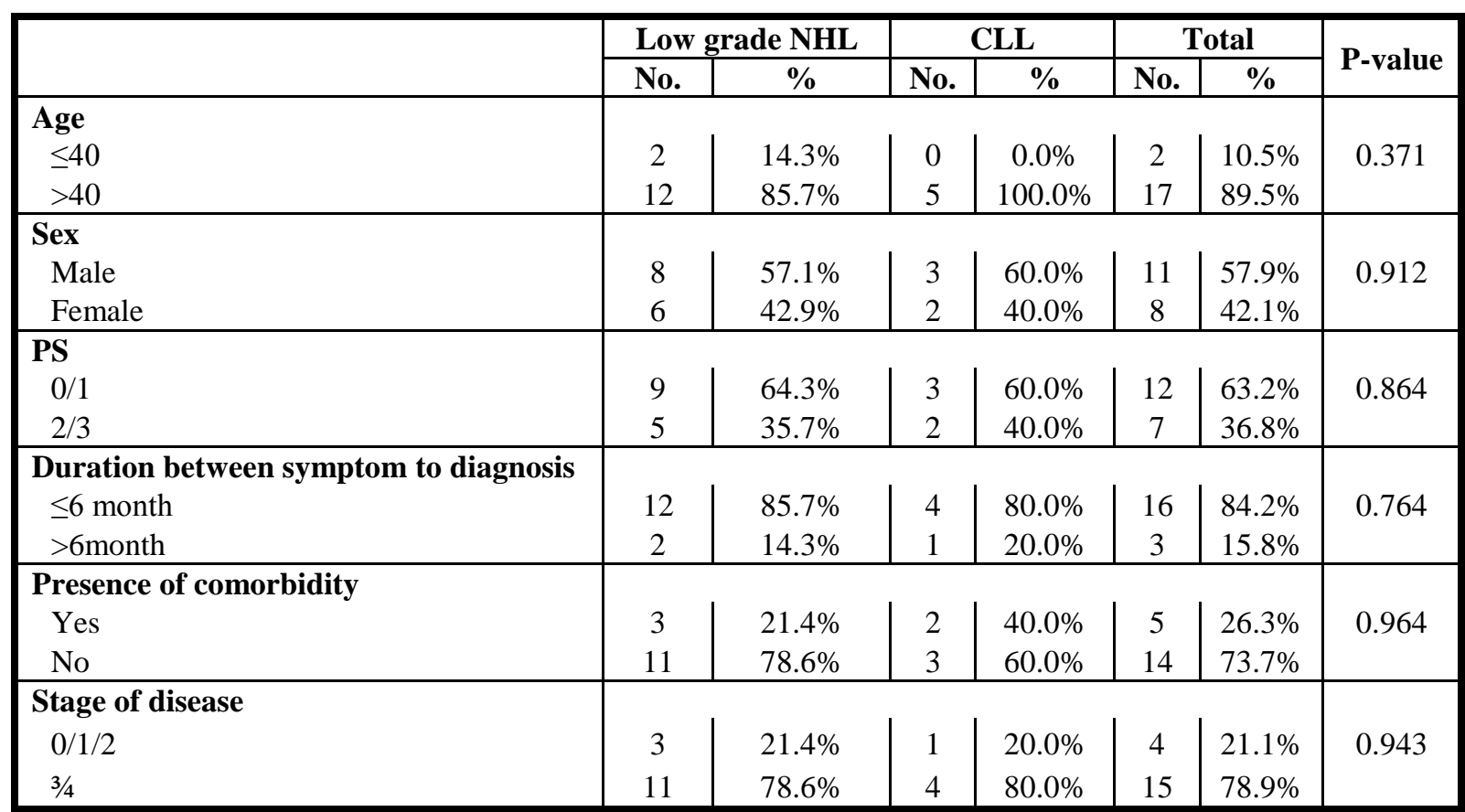

Nineteen patients $(19 / 30-63.4 \%)$ received combination chemotherapy at presentation. The clinical characteristics of these patients. It was noticed that the majority of patients who received chemotherapy had advanced stage of disease.

\section{DISCUSSION}

For indolent lymphomas the goal of therapy has been to maintain the best quality of life and treat only when patients develop symptoms. Any alteration to this approach requires demonstration of improved survival with early institution of therapy, or identification of criteria that define patients sufficiently at "high risk" to merit early therapy. There are many available therapies and no consensus on an optimal first-line or relapse treatment ${ }^{(\mathbf{1 0})}$.

The current work is a retrospective study reviewing the data of patients with confirmed diagnosis of indolent lymphoma referred to us. We conducted this study because we think that the first step to improve the treatment quality should be reviewing the data of our patients and assessing our treatment results with the available drugs at our center.

Follicular lymphoma, the most common subtype of indolent lymphoma, second most common subtype of lymphoma worldwide, accounting for approximately $20 \%$ of malignant lymphomas in adults, but $40 \%$ of all lymphomas diagnosed in the United States and in Western Europe ${ }^{(\mathbf{1 1})}$. In the current study, low grade NHL / CLL represented $0.9 \%$ of all cancer cases and $12.3 \%$ of cases of malignant lymphoma referred to our center during the period of study.

Horning and Rosenberg ${ }^{(12)}$ reported that the median age for indolent lymphoma is 60 years and men and women are equally affected. The current study documented than that reported in the literature. The median age for our patients was around 30 years. This was true for both CLL and low grade lymphoma However, more than half of our patients had the age between 41 and 60 years. The male to female ratio for the whole study group was 1.2: 1.

In asymptomatic patients with indolent forms of NHL, treatment may be deferred until the patient becomes symptomatic as the disease progresses. The National Lymphocare Study identified 471 patients with stage I follicular 
lymphoma. Nonrandomized treatments included radiation therapy (27\%), rituximabchemotherapy (R-chemotherapy) (28\%), watchful waiting (17\%), R-chemotherapy plus radiation therapy $(13 \%)$, and rituximab alone $(12 \%)$. With a median follow-up of 57 months, progression-free survival favored Rchemotherapy or R-chemotherapy plus radiation therapy, but overall survival was nearly identical, all over $90 \%{ }^{(13)}$.

In the current study, only 31 patients were available for treatment assessment. In our study, about $36 \%$ of patients were asymptomatic and were kept under follow up although 36\% (4/11) of them had advanced stage. All but one remained stable for a median period of 16 months $(8-21)$. The remaining patient showed disease progression after 36 months. This figure was higher than that reported by Friedberg et al. ${ }^{(13)}$ although their patients had stage I disease. This may be explained partly by the fact that we include all low grade NHL as well as CLL. However, our patients may different biologic characteristics than those of the western countries. Gribben ${ }^{(10)}$ reported that the clinical course of indolent lymphoma is extremely variable, with some patients having an extremely aggressive course whereas others may live for more than 20 years and never require therapy. More studies with larger number of patients should be conducted to reach firm conclusions

Long-term disease control within radiation fields can be achieved in a significant number of patients with indolent stage I or stage II NHL by using dosages of radiation that usually range from $25 \mathrm{~Gy}$ to $40 \mathrm{~Gy}$ to involved sites or to extended fields that cover adjacent nodal sites. Almost half of all patients treated with radiation therapy alone will relapse out-offield within 10 years ${ }^{(\mathbf{1 4})}$. In the current study, only one patient was treated by irradiation to an retro orbital disease. He was stable with no signs of progression for 26 month till the end of the study.

The alkylating agents chlorambucil and cyclophophamide with or without prednisone and CVP or CHOP, and other alkylator based combination chemotherapy regimens have been the standard of therapy for decades. These combination chemotherapy does not seem to improve OS in older randomized trials and may be responsible for early and late side effects such as a higher incidence of secondary tumors and myelodisplastic syndromes. Rtuximab and other recent target therapy may represent a less toxic systemic treatment alternative to chemotherapy and may finally be leading to improvement in patient survival ${ }^{(\mathbf{1 5})}$.

Rituximab and other target therapy was not available for our patients with low grade NHL / CLL. This was due to financial and administrative reasons. Therefore, in the current study we analyze the treatment results with convential chemotherapy. At our center during the period of study, there was no definitive protocol to follow and the treatment choice depended on physician's preference and availability of drugs.

For low grade NHL, all patients treated by CVP or CHOP showed partial remission on chemotherapy after 4-6 cycles. However, all patients $(100 \%)$ received CVP showed disease progression after a median of 10 months. While, 4 out of the $6(66 \%)$ patients treated by CHOP showed maintained remission for a median period of 16 month. Only one patient $(17 \%$ - 1/6) showed disease progression after 7 month. This shows that CHOP is more effective in achieving maintained response for patients for patients with low grade even if added to rituximab. Luminari et al. ${ }^{(16)}$ concluded that patients initially treated with R-CVP had a higher risk of lymphoma progression compared with those receiving $\mathrm{R}-\mathrm{CHOP}$, as well as a higher risk of requiring additional therapy.

For CLL, all patients treated by CVP or Fludarabine and Cyclophosohamide showed partial remission on chemotherapy after 4-6 cycles. However, all patients $(100 \%)$ received CVP showed disease progression after a median of 12 months. While, 1 out of the $4(25 \%)$ patients treated by Fludarabine and Cyclophosohamide showed maintained remission for a median period of 20 months. This shows that Fludarabine and Cyclophosohamide may be more effective in achieving maintained response for patients with CLL. Thompson et al. ${ }^{(17)}$ reported a 10-year follow-up of 300 patients treated with fludarabine, cyclophosphamide, and rituximab. They showed a sustained PFS in a subset of patients, with 42 patients experiencing no relapses beyond 10.4 years.

There is little or no data regarding the optimal sequencing of treatment approaches in 
indolent lymphoma. Treatment choices remain empiric and should always involve discussion regarding patient choice and goal of therapy. This is likely to become even more complicated because many novel agents are currently being investigated in preclinical and clinical studies, particularly, novel monoclonal antibodies and agents that alter the antiapoptotic pathways. The impact of these new agents on practice will depend on the results of the ongoing clinical trials that should establish a clear-cut treatment approach which leads to cure for the majority of patients.

\section{CONCLUSION}

Radiotherapy One patient treated by involved field irradiation. This patient was 38 years old presented by proptosis. Radiologic examination showed retro orbital mass. There was no lymphoid lesions elsewhere. Biopsy was taken. Histopathologic and immunophenotyping examination confirmed the diagnosis of low-grade NHL. The patient received conformal irradiation 30 Gy over 15 fractions. He showed excellent response on irradiation and kept under follow-up. He was stable and symptom free till the end of study (for 26 months).

Only 31 patients were evaluable for survival analysis with median follow up period of 15 months. Median disease free survival (DFS) was 12 months (95\% CI 0-72.9.

\section{REFERENCES}

\section{Pileri SA, Milani M, Fraternali-Orcioni G} et al. (1998): Classification to the upcoming WHO scheme: a step toward universal categorization of lymphoma entities? Ann Oncol., 9 (6): 607-12.

2. Society for Hematopathology Program (1997): Society for Hematopathology Program. Am J Surg Pathol.;,21 (1): 114-121.

3. Rossi G, Marcheselli L, Dondi A et al. (2015): The use of anthracycline at first-line compared to alkylating agents or nucleoside analogs improves the outcome of salvage treatments after relapse in follicular lymphoma The REFOLL study by the Fondazione Italiana Linfomi Am J Hematol.,90(1):56-61.
4. Tan D, Horning SJ, Hoppe RT et al. (2013): Improvements in observed and relative survival in follicular grade 1-2 lymphoma during 4 decades: the Stanford University experience. Blood, 122(6): 981-987.

5. Schaaf M, Reiser M, Borchmann $P$ et al. (2012): High-dose therapy with autologous stem cell transplantation versus chemotherapy or immunochemotherapy for follicular lymphoma in adults. Cochrane Database Syst Rev.,1: CD007678.

6. Arkozy C, Trneny M, Xerri L et al. (2016): Risk Factors and Outcomes for Patients with Follicular Lymphoma Who Had Histologic Transformation After Response to First-Line Immunochemotherapy in the PRIMA Trial. J Clin Oncol., 34(22): 2575-82.

7. Montoto S, Davies AJ, Matthews J et al. (2007): Risk and clinical implications of transformation of follicular lymphoma to diffuse large B-cell lymphoma. J Clin Oncol., 25 (17): 2426-33.

8. Cheson BD, Trask PC, Gribben JG et al. (2017): Health-related quality of life and symptoms in patients with rituximab-refractory indolent nonHodgkin lymphoma treated in the phase III GADOLIN study with obinutuzumab plus bendamustine versus bendamustine alone. Ann Hematol., 96(2): 253-259.

9. Lunning $M$ and Vose JM (2012): Management of Indolent Lymphoma: Where Are We Now and Where Are We Going. Blood Rev.,26(6): 279288.

10. Gribben JG (2007): How I treat indolent lymphoma. Blood, 109(11):4617-26.

11. Armitage JO (1997): A clinical evaluation of the International Lymphoma Study Group classification of non-Hodgkin's lymphoma. Blood, 89(11):3909-18.

13. Friedberg JW, Byrtek M, Link BK et al. (2012): Effectiveness of first-line management strategies for stage I follicular lymphoma: analysis of the 
National LymphoCare Study. J Clin Oncol., 30 (27): 3368-75.

14. Guckenberger M, Alexandrow N, Flentje $M$ (2012): Radiotherapy alone for stage I-III low grade follicular lymphoma: long-term outcome and comparison of extended field and total nodal irradiation. Radiat Oncol., 7: 103.

15. Hiddemann W, Hoster E, Buske C et al. (2006): Rituximab is the essential treatment modality that underlies the significant improvement in short and long term outcome of patients with advanced stage follicular lymphoma: a 10 year analysis of GLSG trials . Blood, 108:147a.

12. Horning SJ and Rosenberg SA (1984): The natural history of initially untreated low-grade non-Hodgkin's lymphomas. The New England Journal of Medicine, 311(23):1471-1475.

16. Luminari S, Ferrari A, Manni M, Dondi A, Chiarenza A, Merli F, Rusconi C, Tarantino V, Tucci A, Vitolo U, Kovalchuk S (2018): Long-term results of the FOLL05 trial comparing R-CVP versus R-CHOP versus R-FM for the initial treatment of patients with advanced-stage symptomatic follicular lymphoma. J Clin Oncol., 36(7):68996.

17. Thompson PA, Tam CS, O'Brien SM, Wierda WG, Stingo F, Plunkett W, Smith SC, Kantarjian HM, Freireich EJ, Keating MJ (2016): Fludarabine, cyclophosphamide, and rituximab treatment achieves long-term diseasefree survival in IGHV-mutated chronic lymphocytic leukemia. Blood, 127(3):303-9. 